\title{
Electronic band structure, optical, dynamical and thermodynamic properties of cesium chloride $(\mathrm{CsCl})$ from first-principles
}

\author{
Suat Bingol, Bahattin Erdinc*, and Harun Akkus \\ Department of Physics, Faculty of Science, Yuzuncu Yil University, 65080 Van, Turkey
}

Received 3 July 2015 / Accepted 3 November 2015

\begin{abstract}
The geometric structural optimization, electronic band structure, total density of states for valence electrons, density of states for phonons, optical, dynamical, and thermodynamical features of cesium chloride have been investigated by linearized augmented plane wave method using the density functional theory under the generalized gradient approximation. Ground state properties of cesium chloride are studied. The calculated ground state properties are consistent with experimental results. Calculated band structure indicates that the cesium chloride structure has an indirect band gap value of $5.46 \mathrm{eV}$ and is an insulator. From the obtained phonon spectra, the cesium chloride structure is dynamically stable along the various directions in the Brillouin zone. Temperature dependent thermodynamic properties are studied using the harmonic approximation model.
\end{abstract}

Key words: Electronic band structure, Optical properties and dynamical properties, Density functional theory.

\section{Introduction}

To use the materials in nature, their physical and chemical properties must be known. To set information about these properties, we have to determine the electronic structure, optical and dynamical properties of materials. It requires so much effort for calculating electronic band structure and optical properties of crystals. However, in recent years, these properties could be calculated easily using computer calculation based on first-principles. Density functional theory has proved to be highly successful in describing electronic, optical, dynamical and thermodynamic properties of materials. Using density functional theory, many works has been devoted to investigate alkali halides in B2 structure in scientific and technological area because of their interesting electro-optic, electro-mechanic properties and large dielectric constants etc.

The cesium chloride structure has considerable been attracting attention due to its interesting properties. Cesium chloride crystal is classified as an insulator at normal conditions. This compound is interesting due to s-d transition at the conduction band bottom under pressure. At zero pressure, the ideal crystal structure of cesium chloride has a simple cubic lattice, where the cesium atoms are located at the corners of the cube and chloride atom at center.

*e-mail: bahattinerdinc@yyu.edu.tr
The physical properties of cesium chloride are a subject of considerably theoretical and experimental interest. The dielectric constant of cesium chloride was experimentally measured depending on temperature and pressure [1]. Both experimental and theoretical measurements of the second-order Raman spectra of cesium chloride were carried out at both 300 and $80 \mathrm{~K}$ [2]. The ionic conductivity of cesium chloride depending on temperature and hydrostatic pressure were presented and discussed [3]. Spectra for the $\mathrm{Cs}^{+} 3 \mathrm{~d}$ in cesium chloride were investigated in detail [4]. The instability of cesium chloride crystal was investigated under high pressures using a simple model calculation [5]. The electronic band structure and cohesive properties of cesium chloride compound were presented within the local density functional formalism [6]. The preparation and microstructural characterization of cesium chloride were reported in detail using combined cryogenic and room temperature ball milling [7]. The absorption spectra of cesium chloride molecule and dimer were investigated using time offlight technique [8]. The effect of cesium chloride capping layers on semitransparent $\mathrm{Ca} / \mathrm{Ag}$ cathodes was studied [9]. The thermodynamic of cesium chloride crystal between $736 \mathrm{~K}$ and $1077 \mathrm{~K}$ was investigated using Knudsen effusion mass spectrometry [10]. The structural stability of some cesium chloride structure types was studied using full potential method under the density functional theory [11]. First-principles study of the rocksalt-cesium chloride relative phase stability in alkali 
halides was investigated [12]. About 20 alkali halides crystals under hydrostatic pressure were examined the behaviors such as Cohesive and thermal properties, equations of state (EOS), thermodynamic stability ranges of the most common rocksalt (B1) and cesium chloride (B2) phases, phase transition properties and phase transition [13-24].

It is seen from this brief historical search that no a comprehensive calculation of physical properties of cesium chloride crystal from first principles have so far been done or published. In this work, the geometric structural optimization, electronic band structure, total density of valence electrons states, optical properties, dynamical properties, density of phonon states and thermodynamic properties of cesium chloride crystal structure have been examined using a pseudopotential method based on the density functional theory under the generalized gradient approximation.

\section{Discussion and results}

All the parameters of the calculations of electronic band structure, total density of the valence electron states, linear optical properties, dynamical and some thermodynamic properties for cesium chloride compound were performed using ABINIT code [25] based on density functional theory. The norm-conserving generalized gradient approximation pseudopotentials of cesium chloride structure are produced in the Perdew-Burke-Ernzerhof generalized gradient approximation functional (PBE-GGA-96) [26] and used in all calculations. For the electronic wave functions, the plane waves were chosen as the basis set. For solving Kohn-Sham equations [27], the conjugate gradient minimization method [28] was utilized. In all calculations, the exchange-correlation effects were taken into consideration with the Perdew-Burke-Ernzerhof generalized gradient approximation [26]. As the true valence, for cesium atom $6 s$ electron and for chloride atom $3 s$ and $3 p$ electrons were taken. All calculations were performed by taking one molecule per cubic unit cell. The total energy calculations were done to a good convergence at 40 hartree of cutoff energy using $8 \times 8 \times 8$ Monkhorst-Pack mesh grid [29] for geometric structural optimization, electronic band structure, dynamical and thermodynamic properties of cesium chloride crystal structure. However, to calculate optical properties of cesium chloride crystal structure, the irreducible Brillouin zone was sampled with $10 \times 10 \times 10$ Monk-Horst-Pack mesh grid.

Using various methods [30,31], the lattice parameters of cesium chloride crystal structure was investigated. This crystal structure has cubic structure with the space groups $\mathrm{Pm}-3 \mathrm{~m}$. As shown in Table 1, the calculated cubic unit cell has one

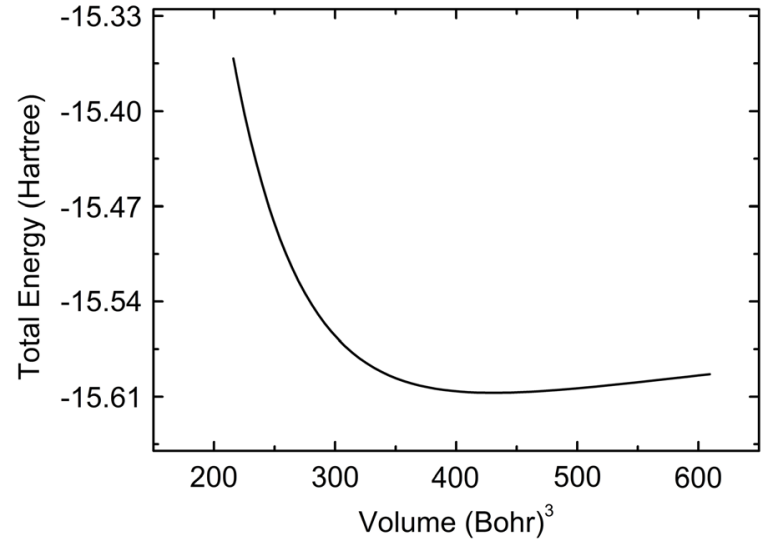

Figure 1. Total energy dependence on volume for cesium chloride.

molecule with two atoms in unit cell. Firstly, the total energy optimization with respect to cutoff energy has been performed. The value of cutoff energy was obtained as 40 hartree. Secondly, the total energy optimization by the number of $k$ points has been performed. The number of $k$ points was calculated as 20 . Then, ground state energy of cubic cesium chloride crystal for different volumes around the experimental equilibrium volume is calculated. The total energy optimization as versus the volume of cesium chloride crystal structure is given in Figure 1. The calculated value of lattice constant is given in Table 1. As seen from Table 1, the theoretically calculated lattice constant value is 7.5540 bohr. In addition, the experimental result and calculated value of lattice constant were compared and given in Table 1. Table 1 indicates that the calculated value for lattice parameters for cesium chloride is $7.5540 \mathrm{bohr}$, while experimental value is $7.7669 \mathrm{bohr}$. As seen, there exists a good agreement between the calculated and experimental results. The obtained lattice constant was used for calculation of all physical properties of this structure.

The physical properties such as the electronic band structure, total density of the valence electron states, optical and dynamical for cesium chloride have been investigated using the ABINIT code. Firstly, pseudopotential method based on the density functional theory within generalized gradient approximation was used in electronic structure calculations of cesium chloride. Electronic band structure and total density of valence electron states of this compound was calculated and plotted in Figure 2. As seen from the figure that the minimum of conduction band and maximum of valence band are located at the $\Gamma$ and $\mathrm{M}$ symmetry points on high symmetry directions of Brillouin zone, respectively. In addition, the obtained band structure of cesium chloride crystal structure has an indirect

Table 1. Crystal structure data of cesium chloride.

\begin{tabular}{llclrr}
\hline & \multicolumn{5}{c}{ Space group Pm-3m (221) } \\
\hline $\begin{array}{l}\text { Cesium } \\
\text { chloride }\end{array}$ & $\begin{array}{c}\text { Calculated } \\
(\text { GGA) }\end{array}$ & Experimental & Atom & $x$ & $y$ \\
\hline$a($ bohr $)$ & 7.5540 & $7.7669[17]$ & Cs & 0.0 & 0.0 \\
$E_{\mathrm{g}}(\mathrm{eV})$ & 5.46 & $8.3[18]$ & $\mathrm{Cl}$ & 0.5 & 0.5 \\
\hline
\end{tabular}


band gap value of $5.46 \mathrm{eV}$ from $\mathrm{M}$ to $\Gamma$ symmetry points of the Brillouin zone. The theoretical band gap of this compound is smaller than the experimental result [31]. It is well known

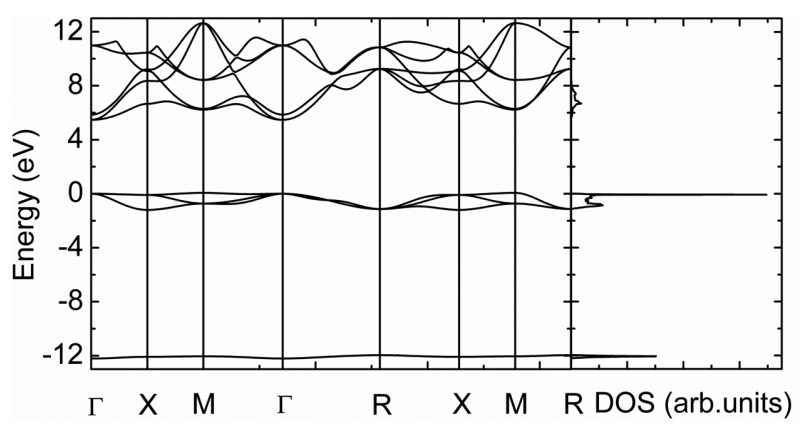

Figure 2. Electronic band structure and total density of states of cesium chloride compound from first-principles.

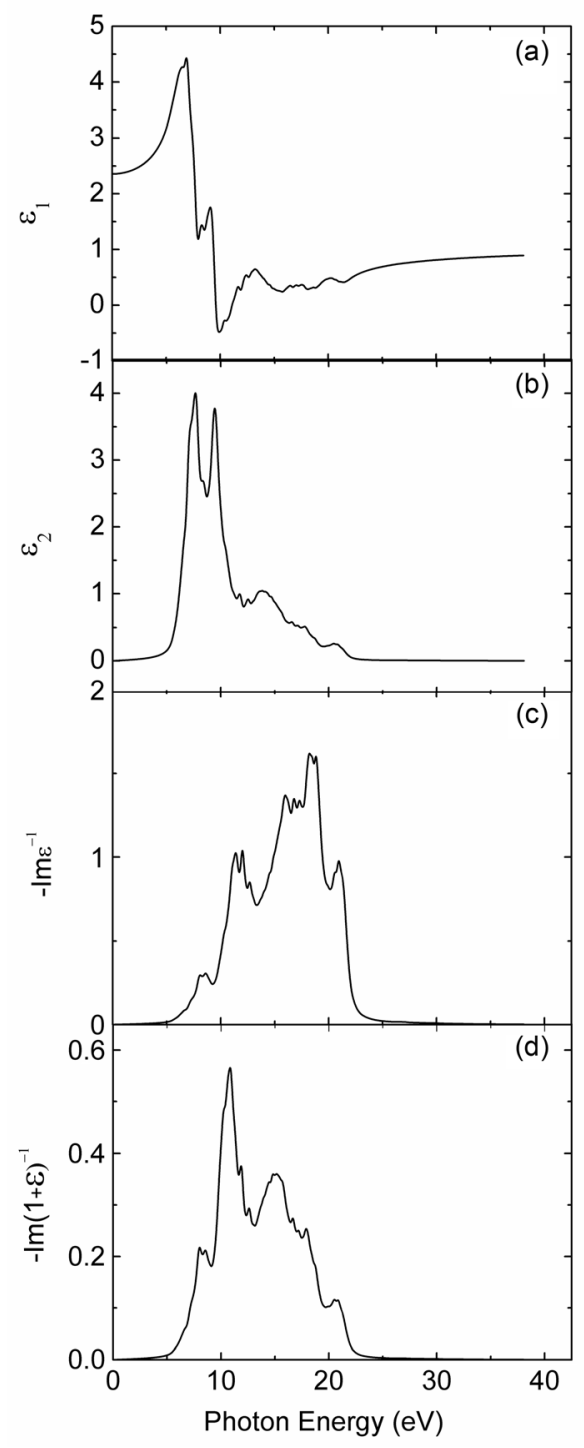

that the obtained band gap by the pseudopotential method is smaller than that obtained from experiments due to imperfection of the method.

Secondly, using the density functional theory within generalized gradient approximation, the real $\left(\varepsilon_{1}\right)$ and imaginary $\left(\varepsilon_{2}\right)$ parts of complex linear dielectric function as a function of the photon energy for cesium chloride are investigated. Moreover, the optical constant such as reflectivity $(R)$, absorption coefficient spectra $(\alpha)$, energy-loss functions for volume $\left(-\operatorname{Im} \varepsilon^{-1}\right)$ and for surface $\left(-\operatorname{Im}(1+\varepsilon)^{-1}\right)$, extinction coefficient $(k)$, refractive index $(n)$ and effective number of electrons $\left(N_{\text {eff }}\right)$ depending on the photon energy of this crystal structure are investigated and given in Figure 3. The calculations only pertain to electronic transitions and neglect all lattice vibrational effects. The calculations of the real and imaginary parts of complex linear dielectric function have been analyzed from 0 to $38 \mathrm{eV}$ photon energy range and plotted in Figures 3a and $3 \mathrm{~b}$. If we analysis the Figure $3 \mathrm{~b}$, the imaginary part of

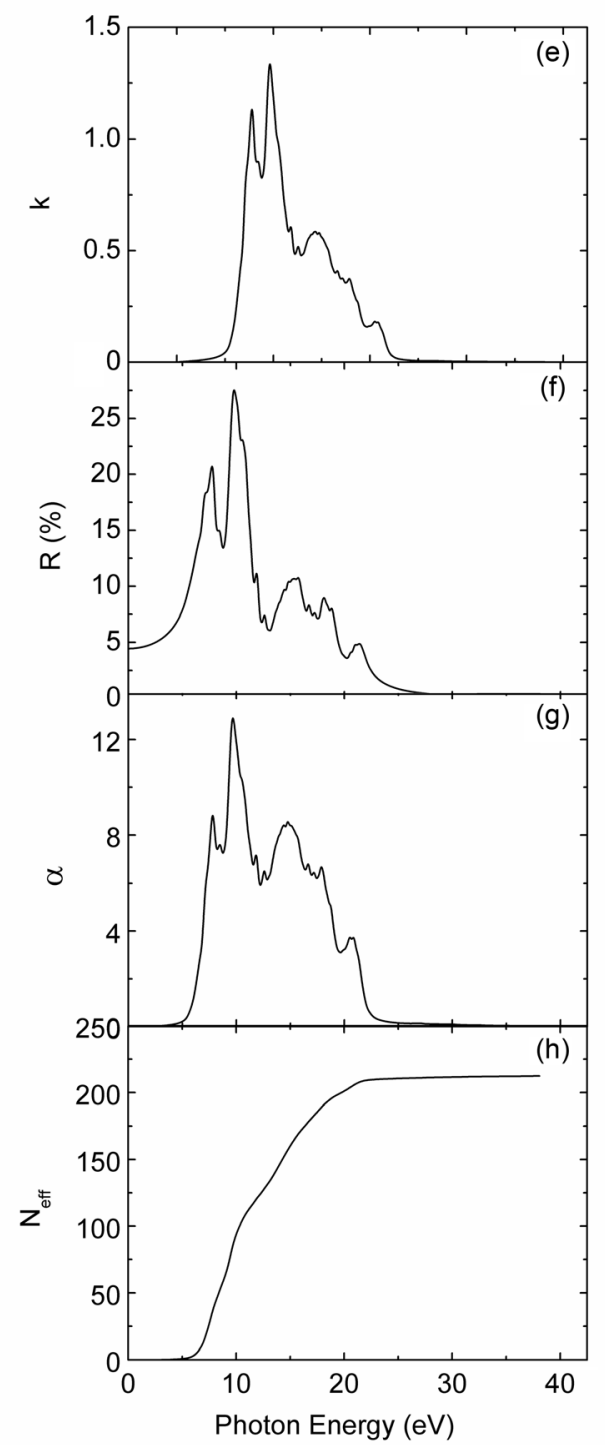

Figure 3. Optical spectra of cesium chloride crystal from first-principles. 
complex linear dielectric function shows peaks which correspond to the transition from valence band to conduction band at many points. In present work, the main peak values in the calculated imaginary part of complex linear dielectric function are at $0.24,4.00,3.78,1.00,0.93,1.05,0.59,0.53$ and 0.28 at $5.28,7.65,9.43,11.68,12.52,13.83,16.56,17.74$ and $20.59 \mathrm{eV}$ of photon energy. There is no absorption between 0 and $5.46 \mathrm{eV}$ photon-energy range. $7.00-10.00 \mathrm{eV}$ energy range is of strong absorption and appreciable reflectivity region. It means that optical absorption increases rapidly in this range. High reflectivity exists between 10.00 and $12.00 \mathrm{eV}$.

Real part of the complex linear dielectric function is presented in Figure 3a. One can see from the figure that the calculated static dielectric constant for cesium chloride crystal structure is 2.37 at $0 \mathrm{eV}$ of photon energy. Figure 3a shows that the real part of complex linear dielectric function is equal to zero at 9.47 and $11.17 \mathrm{eV}$ where the interband transitions consisting mostly of plasmon excitations and the scattering probabilities of volume and surface losses are directly related to the energy loss function. Furthermore, the range of $0-11 \mathrm{eV}$ is classified as a normal dispersion and the range of 11-20 eV are characteristic of an anomalous dispersion.

The optical constants such as energy loss function, reflectivity spectrum, extinction coefficient, absorption coefficient and effective number of electrons were investigated using complex linear dielectric function. The energy-loss functions describing the energy loss of fast electrons making transitions in the material for volume and surface are plotted in Figures $3 \mathrm{c}$ and $3 \mathrm{~d}$, respectively. The calculated energy-loss functions for volume and surface show mainly intense maxima as presented in Figures 3c and 3d, respectively. Here, the obtained main peak values for volume loss function are at $0.3,1.03,1.04$, $1.37,1.62$ and 0.98 at $8.30,11.42,12.05,15.92,18.20$ and $20.90 \mathrm{eV}$ of photon energy, while the obtained main peak values for surface loss function are at $0.22,0.57,0.36,0.25$ and 0.12 at $7.94,10.76,15.04,17.94$ and $20.67 \mathrm{eV}$ of photon energy. These peak values coincide with one of zeros of linear dielectric function's real part. The sharp maxima in the energyloss functions are related to plasma oscillations. The calculated extinction coefficient, reflectivity spectrum and absorption coefficient show mainly intense maxima as presented in Figures $3 \mathrm{e}-3 \mathrm{~g}$, respectively. Here, the obtained main peak values for extinction coefficient are at 1.13, 1.34, 0.60, 0.37 and 0.18 at $7.76,9.68,14.41,17.84$ and $20.57 \mathrm{eV}$ of photon energy. The obtained main peak values for reflectivity spectrum are at $0.21,0.28,0.11,0.089$ and 0.049 at $7.66,9.81,15.42,18.12$ and $21.37 \mathrm{eV}$ of photon energy. The obtained main peak values for absorption coefficient are at 8.77, 12.92, 8.56, 6.69 and 3.73 at $7.82,9.69,14.67,17.87$ and $20.67 \mathrm{eV}$ of photon energy. The effective number of valence electron which contributes to the interband transition is investigated using the sum rule [32]. This calculation is given in Figure $3 \mathrm{~h}$. This figure indicates that the effective number of valence electron of cesium chloride crystal structure is zero up to $5.46 \mathrm{eV}$, then rises rapidly up to $20 \mathrm{eV}$ and reaches a saturation value above $25.0 \mathrm{eV}$.

The calculated refractive index of cesium chloride over a wide range of wavelengths is shown in Figure 4. It is seen from Figure 4 that the calculated value of refractive index decreases with a transition from the intrinsic absorption region to long waves when normal dispersion takes place. A maximum value of the refractive index as a function of wavelength was obtained as a value of 2.2 .

In the unit cell of cesium chloride crystal contains two atoms. The cesium atom is located at the point of $(0.0,0.0,0.0)$ which chloride atom at the point of $(0.5,0.5,0.5)$ in the reduced coordinates inside the unit cell. Using the generalized gradient approximation, the phonon properties of cesium chloride were investigated. The calculated phonon dispersion curves and phonon density of states of cesium chloride crystal along the high-symmetry directions are plotted in Figure 5. Six phonon branches totally appear due to two atoms per unit cell for cesium chloride crystal. While three of them are the acoustical branches, the remaining are the optical branches. This figure shows that this structure is dynamically stable because all phonon frequencies are positive. Because of differences in masses of cesium and chloride atoms, there is a gap between the acoustical and the optical phonon branches as expected. The band gap between the acoustic and optical branches is about $1.2 \mathrm{THz}$. The frequency values of longitudinal and transverse optical phonon modes in the center of Brillouin zone are calculated as $5.45 \mathrm{THz}$ and $3.96 \mathrm{THz}$, respectively. Some of the acoustical and optical branches are degenerate along the symmetrical directions

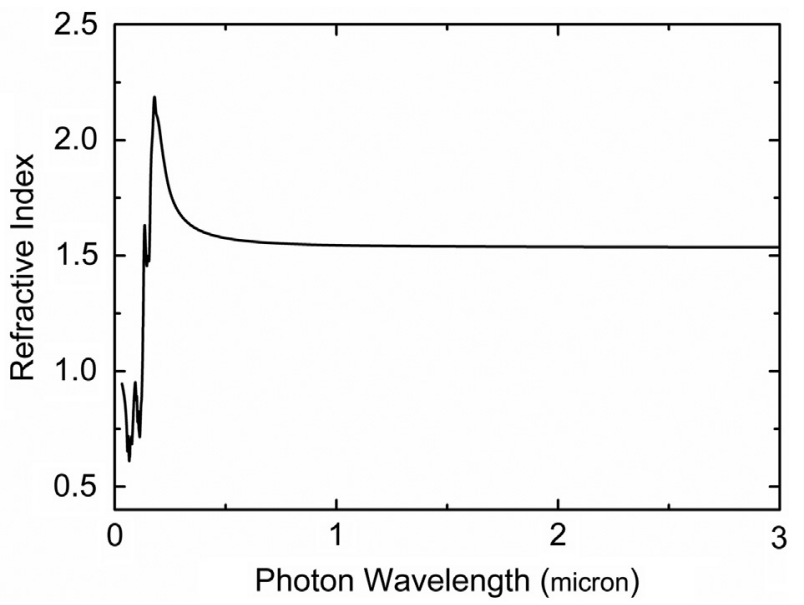

Figure 4. Refractive index dispersion of cesium chloride compound from first-principles.

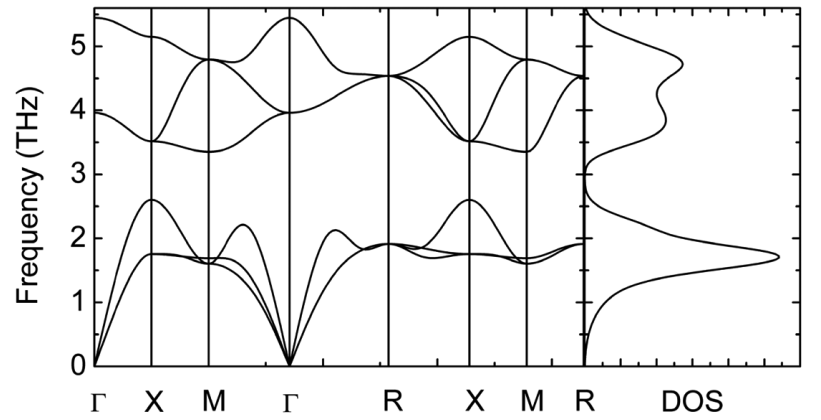

Figure 5. Dispersion curves for phonons and phonon density of states of cesium chloride crystal from first-principles. 

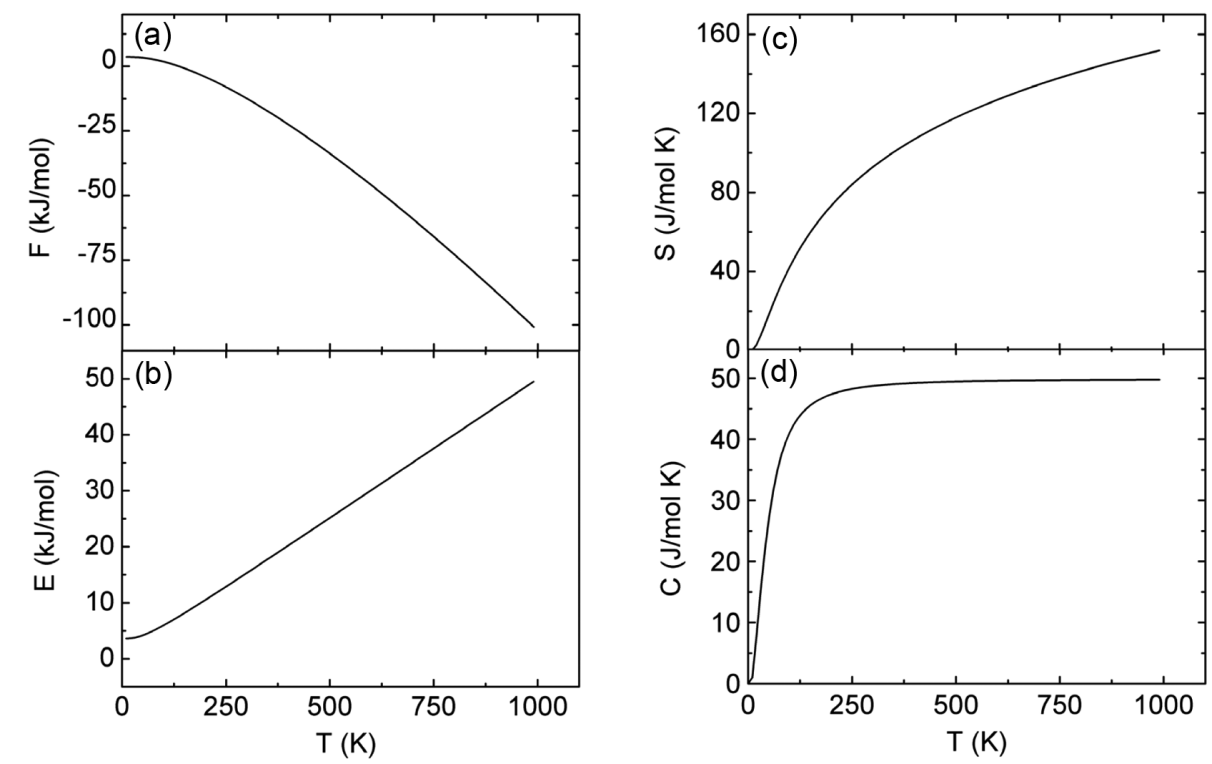

Figure 6. Thermodynamic properties of cesium chloride crystal from first-principles. (a) Contribution of phonons to free energy as a function of temperature, (b) phonon contribution to internal energy as a function of temperature, (c) the entropy as a function of temperature, and (d) specific heat as a function of temperature.

inside the Brillouin zone. As see in Figure 5, the direction from $\Gamma$ to $\mathrm{R}$ and from $\Gamma$ to $\mathrm{X}$ is more symmetrical than the other directions. Transverse acoustical and transverse optical phonon modes are degenerate along these symmetry directions. When the symmetry is broken, the degeneracy in the modes along the other directions disappears. In high symmetry directions of $\Gamma$ to $\mathrm{R}$ and $\Gamma$ to $\mathrm{X}$, acoustical dispersion curves for phonons are the linear as a function of $k$ for small values of $k$ as seen in Figure 5. The phonon density of states is shown in the rightmost panel of Figure 5. While transverse acoustic phonon modes are between $0.3 \mathrm{THz}$ and $1.6 \mathrm{THz}$ in the phonon density of states, longitudinal acoustical phonon modes lie to between $1.6 \mathrm{THz}$ and $2.6 \mathrm{THz}$ in the phonon density of states. All the optical phonon modes are between $3.5 \mathrm{THz}$ and $5.5 \mathrm{THz}$ in the phonon density of states.

Thermodynamic properties of cesium chloride crystal have been investigated using whole phonon band structure calculations. The phonon contributions to the Helmholtz free energy $(F)$ and internal energy $(E)$, the entropy $(S)$ and the specific heat $\left(C_{\mathrm{v}}\right)$ of cesium chloride crystal as a function of temperature are calculated. These properties as a function of temperature are plotted in Figure 6 . The thermodynamic properties of this crystal are investigated using the harmonic approximation [33]. As seen from Figures 6a and 6b, the contribution of phonons to the internal energy and free energy do not vanish at zero temperature. At zero temperature, the contributions of phonons to the internal and free energies are same and equal to $3.8 \mathrm{~kJ} / \mathrm{mol}$. As seen from Figure $6 \mathrm{a}-6 \mathrm{c}$, while the free energy of cesium chloride crystal decreases, as the temperature rises, internal energy and entropy increase. The specific heat is given in Figure 6d. The specific heat reaches a classic limit value at $830 \mathrm{~K}$.

\section{Conclusions}

As far as we know, the structure optimization, electronic band structure, density of states, optical, dynamic and thermodynamic properties of cesium chloride have not been investigated theoretically. In the present work, by linearized augmented plane wave method using the density functional theory under the generalized gradient approximation, the geometric structural optimization, electronic band structure, total density of valence electron state, optical properties, density of phonon state, dynamical and thermodynamical properties of cesium chloride crystal structure have been investigated and discussed in detail. The calculated structure optimization of cesium chloride structure was compared with the experimental result and a good agreement between them was found. As a result of the calculations, it is seen that the fundamental gap of cesium chloride crystal structure is indirect one from $\mathrm{M}$ to $\Gamma$ in the Brillouin zone. The found band gap is smaller than the experimental one. The real and imaginary parts of complex linear dielectric function have been investigated as a function of the photon energy. The obtained phonon spectrum of cesium chloride crystal structure indicates that this crystal is dynamically stable along the various directions in the Brillouin zone. Moreover, the temperature dependent thermodynamic properties such as Helmholtz free energy, internal energy, entropy and heat capacity are investigated using the harmonic approximation model after calculating the phonon frequencies. The detailed definition of these properties is given.

Acknowledgements. This work has been supported by The Unit of Scientific Research Projects of Yuzuncu Yil University under project No. 2011-FED-B010. 


\section{References}

1. Havinga EE, Bosman AJ. 1965. Temperature dependence of dielectric constants of crystals with $\mathrm{NaCl}$ and $\mathrm{CsCl}$ structure. Phys. Rev., 140, A292-A303.

2. Agrawal BS, Kirby RD, Hardy JR. 1975. Theoretical and experimental studies of the second-order Raman spectra of $\mathrm{CsCl}, \mathrm{CsBr}$, and CsI. Phys. Rev. B, 11, 5153-5162.

3. Samara A. 1980. Pressure and temperature dependences of the ionic conductivity of CsCl. Phys. Rev. B, 22, 6476-6479.

4. Motais P, Belin E, Bonnelle C. 1982. Direct radiative recombination of core-hole highly excited states in $\mathrm{CsCl}$. Phys. Rev. B, 25, 5492-5498.

5. Vohra YK, Duclos SJ, Ruoff AL. 1985. Instability of the CsC1 Structure in ionic solids at high pressures. Phys. Rev. Lett., 54, 570-573.

6. Satpathy S. 1986. Electron energy bands and cohesive properties of CsC1, CsBr, and Csl. Phys. Rev. B, 33, 8706-8715.

7. Tiwary CS, Verma A, Biswas K, Mondal AK, Chattopadhyay K. 2011. Preparation of ultrafine $\mathrm{CsCl}$ crystallites by combined cryogenic and room temperature ball milling. Ceram. Int., 37, 3677-3686.

8. Ruusa R, Kukk E, Huttula M, Aksela H, Aksela S. 2005. Resonant VUV absorption in $\mathrm{CsCl}$ monomers and dimersexperiment and theory. J. Electron Spectros. Relat. Phenomena, 144-147, 1215-1218.

9. Leea CJ, Hana JI, Choib DK, Moonc DG. 2010. Transparent organic light-emitting devices with $\mathrm{CsCl}$ capping layers on semitransparent $\mathrm{Ca} / \mathrm{Ag}$ cathodes. J. Mater. Sci. Eng. B, 172, 76-79.

10. Lisek I, Kapala J, Miller M. 1998. Thermodynamic study of the $\mathrm{CsCl}-\mathrm{NdCl}_{3}$ system by Knudsen effusion mass spectrometry. J. Alloys Compd., 278, 113-122.

11. Novakovic $\mathrm{N}$, Ivanovic $\mathrm{N}$, Koteski $\mathrm{V}$, Radisavljevic I, Belosevic-Cavor J, Cekic B. 2006. Structural stability of some $\mathrm{CsCl}$ structure HfTM $(\mathrm{TM}=\mathrm{Co}, \mathrm{Rh}, \mathrm{Ru}, \mathrm{Fe})$ compounds. Intermetallics, 14, 1403-1410.

12. Florez M, Recio JM, Francisco E, Blanco MA, Martin Pendas A. 2002. First-principles study of the rocksalt-cesium chloride relative phase stability in alkali halides. Phys. Rev. B, 66, 144112-144117.

13. Born M, Huang K. 1954. Dynamical theory of crystal lattices. Oxford University Press: New York.

14. Darnell AJ, McCollum WA. 1970. Thermodynamics of the $\mathrm{Fm} 3 \mathrm{~m} \rightleftarrows \mathrm{Pm} 3 \mathrm{~m}$ transition in the potassium and rubidium halides. J. Phys. Chem. Solids, 31, 805-815.

15. Basset WA, Takahashi T, Mao HK, Weaber JS. 1968. Pressureinduced phase transformation in NaCl. J. Appl. Phys., 39, 319325.

16. Petrunina TI, Soshnikov VI, Estrin EI. 1972. Hysteresis and the features of phase transformation in rubidium halides under pressure. Sov. Phys. Crystallogr., 17, 367-369.
17. Vaidya N, Kennedy GC. 1971. Compressibility of 27 halides to 45 kbar. J. Phys. Chem. Solids, 32, 951-964.

18. Mei WN, Boyer LL, Mehl MJ, Ossowski MM, Stokes HT. 2000. Calculation of electronic, structural, and vibrational properties in alkali halides using a density-functional method with localized densities. Phys. Rev. B, 61, 11425-11431.

19. Lacam A, Peyronneau J. 1973. Polymorphic transition of rubidium chloride under high pressure. Effect of experimental parameters on hysteresis effect. J. Phys. (Paris), 34, 1047-1053.

20. Sims CE, Barrera GD, Allan NL, Mackrodt WC. 1998. Thermodynamics and mechanism of the B1-B2 phase transition in group-I halides and group-II oxides. Phys. Rev. B, 57, 11164-11172.

21. Pistorius CWFT. 1976. Phase relations and structures of solids at high pressures. Prog. Solid State Chem., 11, 1-151.

22. Kohler U, Johannsen PG, Hozapfel WB. 1997. Equation-ofstate data for CsCl-type alkali halides. J. Phys. Condens. Matter, 9, 5581-5592.

23. Yagi T. 1978. Experimental determination of thermal expansivity of several alkali halides at high pressures. J. Phys. Chem. Solids, 39, 563-571.

24. Hofmeister AM. 1997. IR spectroscopy of alkali halides at very high pressures: Calculation of equations of state and of the response of bulk moduli to the B1-B2 phase transition. Phys. Rev. B, 56, 5835-5855.

25. Gonze X, Beuken JM, Caracas R, Detraux F, Fuchs M, Rignanese GM, Sindic L, Verstrate M, Zerah G, Jollet F, Torrent M, Roy A, Mikami M, Ghosez P, Raty JY, Allan DC. 2002. First-principle computation of material properties: the ABINIT software. Comput. Mater. Sci., 25, 478-492.

26. Perdew JP, Burke K, Ernzerhof M. 1996. Generalized gradient approximation made simple. Phys. Rev. Lett., 77, 3865-3868.

27. Kohn W, Sham LJ. 1965. Self-consistent equations including exchange and correlation effects. Phys. Rev., 140, A1133.

28. Payne MC, Teter MP, Allan DC, Arias TA, Joannopoulos JD. 1992. Iterative minimization techniques for ab initio totalenergy calculations: molecular dynamics and conjugate gradients. Rev. Mod. Phys., 64, 1045-1097.

29. Monkhorst HJ, Pack JD. 1976. Special points for Brillouin-zone integrations. Phys. Rev. B, 13, 5188-5192.

30. Kittel C. 1996. Introduction to solid state physics, 7th Edition. John Wiley and Sons Inc., USA.

31. Poole RT, Jenkin JG, Liesegang J, Leckey RCG. 1975. Electronic band structure of the alkali halides. I. Experimental parameters. Phys. Rev. B, 11, 5179-5185.

32. Philipp HR, Ehrenreich H. 1963. Optical properties of semiconductors. Phys. Rev., 129, 1550-1560.

33. Lee C, Gonze X. 1995. Ab initio calculation of the thermodynamic properties and atomic temperature factors of $\mathrm{SiO}_{2} \alpha$ quartz and stishovite. Phys. Rev. B, 51, 8610-8163.

Cite this article as: Bingol S, Erdinc B \& Akkus H: Electronic band structure, optical, dynamical and thermodynamic properties of cesium chloride $(\mathrm{CsCl})$ from first-principles. Int. J. Simul. Multisci. Des. Optim., 2015, 6, A7. 